\title{
How Accurate are ECG Parameters from Wearable Single-lead ECG System for 24-hours Monitoring
}

\author{
Zhipeng Cai ${ }^{1}$, Jianqing $\mathrm{Li}^{1,2}$, Xiangyu Zhang ${ }^{1}$, Qin Shen ${ }^{3}$, Alan Murray ${ }^{4}$ and Chengyu Liu ${ }^{1}$ \\ ${ }^{1}$ School of Instrument Science and Engineering, Southeast University, Nanjing, China \\ ${ }^{2}$ School of Biomedical Engineering and Informatics, Nanjing Medical University, Nanjing, China \\ ${ }^{3}$ The First Affiliated Hospital of Nanjing Medical University, Nanjing, China \\ ${ }^{4}$ School of Engineering, Newcastle University, Newcastle upon Tyne, UK
}

\begin{abstract}
Wearable electrocardiogram (ECG) devices have been quickly developed for convenient long-term monitoring. To further verify the accuracy of textile electrode based longterm wearable ECG analysis, a wearable ECG device was used to record 24-hours long-term ECGs simultaneously with a Holter monitor. Clinical parameters were derived from the wearable ECGs, and were compared with the reports from the Holter monitor. Specifically, ECG parameters of the measured total time, the beat number, the slowest heart rate, the mean heart rate, the fastest heart rate, the beat number of tachycardia, the beat number of bradycardias, Heart rate variability (HRV) parameters of SDNN, SDANN, RMSSD, PNN50, as well as the detection of premature atrial contraction (PAC) and premature ventricular contraction $(P V C)$, were analysed and compared. Mean relative errors (MREs) of ECG parameters between the wearable ECG analysis and Holter report were all less than $10 \%$ except the times of bradycardias (13.97\%). MREs for HRV parameters were all less than $14 \%$, and MREs for counting premature atrial contraction (PAC) and premature ventricular contraction $(P V C)$ were 61.60 and $395.95 \%$, respectively. The results showed that ECG and HRV parameters from wearable ECGs were comparable to the Holter monitor, while there was large bias for PAC and PVC detection.
\end{abstract}

\section{Introduction}

Cardiovascular disease remains a leading cause of death worldwide, accounting for $31 \%$ of all deaths each year [1]. In practice, Holter monitors are a common device for dynamic long-term electrocardiogram (ECG) monitoring (usually 24-48 hours). However, Holter monitors cannot alert in real time, and their bulky nature may cause activity constraints and discomfort. Recent advances in wearable technology and Internet of Things (IoT) devices have facilitated remote, continuous and convenient ECG monitoring [2]. Several wearable ECG monitoring devices have been developed, and they are reported to be able to obtain ECG morphology that can comparable with Holter monitors [3-5]. The dry electrodes they employed can provide more comfort to wearer, and would introduce more baseline wander and motion noise during daily activities. This challenge has prompted researchers to develop new methods for wearable ECG processing, even wearable ECG diagnostics, to reduce the manual interpretation work for physicians [6].

In recent years, numerous algorithms have been developed for the automatic, computer-based and accurate recognition of arrhythmias in an ECG recorder [7-9]. Lee et.al. developed a smart ECG patch for wearable ECG monitoring, and the sensitivity (Se) and positive predictive $(+\mathrm{P})$ value of the built-in R-peak detection algorithm are $99.29 \%$ and $100 \%$, respectively [7], the system can also perform well in long-term heart rate variability (HRV) analysis. Chen et.al. proposed a real-time QRS detection and $\mathrm{R}$ point recognition method for a wearable single-lead ECG device, which can achieve a $99.82 \%$ Se and $99.81 \%$ $+\mathrm{P}$ on MIT-BIH database [8]. Rienzo et.al. compared the detected cardiac rhythm and arrhythmic events from ECG signals recorded by MagIC, a textile-based wearable system, and traditional ECG device, the results indicated that the performance of MagIC was comparable to traditional ECG device in static condition [9]. All the methods can achieve a satisfactory result for wearable ECG analysis and classification. However, these methods are all based on short time ECG monitoring, the accuracy of wearable ECG analysis and recognition in long-term ECG recordings still requires research.

In this paper, the ECG signals were synchronously recorded by a Holter and a wearable ECG system. Thereafter, the standard ECG parameters derived from wearable ECG signals were compared with those from Holter reports to investigate the accuracy of ECG parameters derived from wearable ECG signals.

\section{Methods}

In order to investigate the accuracy of ECG parameters 
from wearable single-lead ECG system for 24 hours monitoring, the ECG recordings were measured by a Holter and a wearable ECG system, synchronously. The lead II wearable ECG recordings were adopted and processed to calculate the ECG parameters and the derived parameters were compared with Holter reports (shown in Fig. 1).

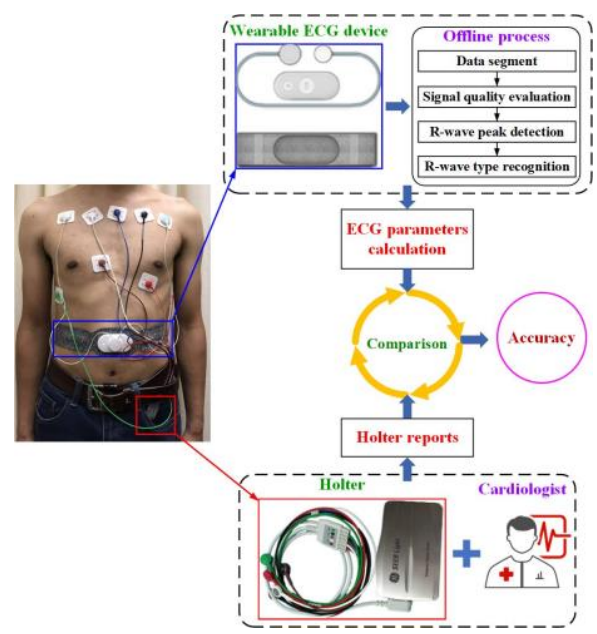

Figure 1. The structure of this study.

\subsection{ECG monitoring}

The Holter used in this study was SEER Light (General Electric Company Ltd. http://www.ge.com), it could realize 24-hour dynamic ECG monitoring (Sampling frequency: $1000 \mathrm{~Hz}$, sensitivity: $10 \mathrm{~mm} / \mathrm{mV}$ ), and provide a standard Holter reports after monitoring. The Holter reports were rechecked and corrected by a cardiologist.

The wearable ECGs were collected using conductive textile dry electrodes embedded in the Wearable 12-lead ECG SmartVest system [2]. A self-charged ECG module was embedded in the back of SmartVest, which could startup signal recording, and implement hardware filtering, denoising and amplifying (frequency band $=0.05$ to $125 \mathrm{~Hz}$, gain=400). The ECG was sampled at $400 \mathrm{~Hz}$, and was stored locally in the memory card in ECG module, being transmitted to a connected smartphone via Bluetooth.

Ten volunteers aged 39 to 81 participated in this study, and two of them have a history of premature contractions (volunteer 2 and 7). The recording time length of each volunteer was about 22 hours.

\subsection{Wearable ECG signal processing}

Considering the real-time requirement of wearable ECG system, the recorded wearable ECG signals were segmented into 10 -s segments with 2-s overlap to simulate the real-time condition. Then, the signal quality of each 10s segment was evaluated firstly, and the segments with large noise or lead shedding were excluded. Secondly, the R-wave peaks of each segment were detected using the wavelet transform modulus maxima method $[10,11]$. Next, the quasi-premature beat $(\mathrm{qPB})$ were recognized based on several rules derived from template matching and RR interval information; then, the results were refined by rejecting false positive $\mathrm{PAC}, \mathrm{PVC}$ and $\mathrm{N}$ in $\mathrm{qPB}$. The refined rules were also based on template matching and $\mathrm{RR}$ interval information. After all segments were processed, the results of $\mathrm{R}$-wave peaks and R-wave types were combined to obtain the total peaks and types of each recording. Finally, the chosen ECG parameters were calculated based on the total peaks and types.

\subsection{ECG parameters}

Referring to the ECG parameters demonstrated in Holter reports, thirteen parameters were compared in this study (shown in Table 1).

Table 1. The adopted ECG parameters and their abbreviations.

\begin{tabular}{|c|c|c|}
\hline ECG parameters & & Abbreviation \\
\hline \multirow{7}{*}{ Rhythm parameters } & Total time & - \\
\hline & Beat number & $\mathrm{BN}$ \\
\hline & Mean heart rate & $\mathrm{mHR}$ \\
\hline & Fastest heart rate & fHR \\
\hline & Slowest heart rate & sHR \\
\hline & Times of tachycardia & $\mathrm{Tt}$ \\
\hline & Times of bradycardias & $\mathrm{Tb}$ \\
\hline \multirow{4}{*}{ HRV parameters } & SDNN & - \\
\hline & SDANN & - \\
\hline & RMSSD & - \\
\hline & PNN50 & - \\
\hline \multirow{2}{*}{$\begin{array}{l}\text { Abnormal } \\
\text { recognition }\end{array}$} & $\begin{array}{l}\text { Premature atrial } \\
\text { contraction number }\end{array}$ & PAC \\
\hline & $\begin{array}{l}\text { Premature ventricular } \\
\text { contraction number }\end{array}$ & PVC \\
\hline
\end{tabular}

\section{Experiments and results}

The volunteers were required to wear the Holter and wearable ECG system in the First Affiliated Hospital of Nanjing Medical University under the help of medical staff. After all the configuration implemented, the long-term ECG data during daily activities were collected outside the hospital without any supervision. After 24-hour recording, the volunteers returned the Holter and wearable ECG system to the hospital. The ECG data was downloaded from the cloud, and processed off-line. The compared ECG parameters were illustrated in Table 2.

From Table 2, we can see that the minimal recording time from wearable ECG is in volunteer 4 , which is 4 hours less than from Holter. Meanwhile, it also leads to the detected beat number of this volunteer is the minimum, and the relative error is $25.76 \%$. The mHR from Holter are all larger than from the wearable ECG system, the maximal relative error is 8.77 in volunteer 4 . The difference of fHR fluctuated drastically, it can be $44.91 \%$ larger from Holter to the wearable ECG system. The sHR values are approximate, the maximal relative error is $18.75 \%$ in volunteer 8. The $\mathrm{Tt}$ from Holter is 101 in volunteer 6, which is more than two times from the wearable ECG system. The severe fluctuation is obvious in $\mathrm{Tb}$, and the maximal relative error occurs in volunteer $2(22.49 \%)$. For 
$\mathrm{HRV}$, the most pronounced relative error of SDNN is $8.04 \%$ in volunteer 5 . The maximal relative error of SDANN is $7.81 \%$ in volunteer 7 , and reaches to $30 \%$ for RMSSD in volunteer 6. For PNN50, the difference is significant in volunteer 8 , but the maximal relative error is $22.22 \%$ in volunteer 9. From the perspective of $\mathrm{PB}$ detection, the maximal PAC and PVC error is in volunteer 3 and 8, the relative error are 115.79 and $3133.33 \%$, respectively.

Fig 2 (a) illustrates the ratio of mean values for each ECG parameter from Holter to wearable ECG. It can be seen that the almost all the ECG parameters from wearable ECG recordings are consistent with those from Holter reports, except PAC and PVC numbers. The mean relative errors (MREs) of the ten volunteers from Holter reports and wearable ECG parameters were shown in Fig 2 (b). The MREs for rhythm parameters and HRV parameters are all less than $14 \%$, while for counting premature atrial contraction (PAC) and premature ventricular contraction (PVC) were 61.60 and $395.95 \%$, respectively. The results indicate that ECG and HRV parameters from wearable Table 2. The comparison of ECG parameters between Holter reports and the wearable ECG system.

\begin{tabular}{|c|c|c|c|c|c|c|c|c|c|c|c|}
\hline Patient ID & & 1 & 2 & 3 & 4 & 5 & 6 & 7 & 8 & 9 & 10 \\
\hline \multirow{3}{*}{$\begin{array}{l}\text { Total time } \\
\text { (h) }\end{array}$} & Holter & 22.3 & 21.67 & 21.68 & 22.05 & 22.92 & 23 & 21.87 & 22.1 & 22.75 & 23 \\
\hline & Our & 24.78 & 24.49 & 23.12 & 17.73 & 23.87 & 23.41 & 23.09 & 22.32 & 24.18 & 22.81 \\
\hline & Relative error (\%) & 11.12 & 13.01 & 6.64 & 19.59 & 4.14 & 1.78 & 5.58 & 1.00 & 6.29 & 0.83 \\
\hline \multirow[t]{3}{*}{ Beat number } & Holter & 98,658 & 89,714 & 87,839 & 73,758 & 75,348 & 92,049 & 90,863 & 69,178 & 90,162 & 88,955 \\
\hline & Our & 106,322 & 97,769 & 92,260 & 54,757 & 75,754 & 86,811 & 89,779 & 63,751 & 94,954 & 87,476 \\
\hline & Relative error (\%) & 7.77 & 8.98 & 5.03 & 25.76 & 0.54 & 5.69 & 1.19 & 7.84 & 5.31 & 1.66 \\
\hline \multirow{3}{*}{$\begin{array}{l}\text { Mean HR } \\
\text { (beats/min) }\end{array}$} & Holter & 74 & 68 & 68 & 57 & 55 & 68 & 66 & 52 & 67 & 66 \\
\hline & Our & 72 & 65 & 65 & 52 & 53 & 63 & 63 & 48 & 66 & 64 \\
\hline & Relative error $(\%)$ & 2.70 & 4.41 & 4.41 & 8.77 & 3.64 & 7.35 & 4.55 & 7.69 & 1.49 & 3.03 \\
\hline \multirow{3}{*}{$\begin{array}{l}\text { Fastest HR } \\
\text { (beats/min) }\end{array}$} & Holter & 94 & 95 & 112 & 97 & 82 & 167 & 92 & 93 & 91 & 98 \\
\hline & Our & 93 & 110 & 111 & 95 & 77 & 92 & 104 & 89 & 92 & 98 \\
\hline & Relative error $(\%)$ & 1.06 & 15.79 & 0.89 & 2.06 & 6.10 & 44.91 & 13.04 & 4.30 & 1.10 & 0.00 \\
\hline \multirow{3}{*}{$\begin{array}{l}\text { Slowest HR } \\
\text { (beats/min) }\end{array}$} & Holter & 60 & 53 & 45 & 36 & 44 & 48 & 44 & 32 & 51 & 53 \\
\hline & Our & 59 & 54 & 47 & 40 & 44 & 49 & 49 & 38 & 51 & 54 \\
\hline & Relative error (\%) & 1.67 & 1.89 & 4.44 & 11.11 & 0.00 & 2.08 & 11.36 & 18.75 & 0.00 & 1.89 \\
\hline \multirow{3}{*}{$\begin{array}{l}\text { Times of } \\
\text { tachycardia }\end{array}$} & Holter & 0 & 0 & 496 & 0 & 0 & 101 & 0 & 0 & 0 & 0 \\
\hline & Our & 0 & 0 & 489 & 0 & 0 & 46 & 0 & 0 & 0 & 0 \\
\hline & Relative error (\%) & 0.00 & 0.00 & 1.41 & 0.00 & 0.00 & 54.46 & 0.00 & 0.00 & 0.00 & 0.00 \\
\hline \multirow{3}{*}{$\begin{array}{l}\text { Times of } \\
\text { bradycardias }\end{array}$} & Holter & 14 & 4,313 & 25,940 & 45,071 & 60,962 & 21,833 & 14,498 & 60,312 & 23,683 & 31,826 \\
\hline & Our & 13 & 3,343 & 22,282 & 35,454 & 53,581 & 17,388 & 16,199 & 54,315 & 21,670 & 28,006 \\
\hline & Relative error (\%) & 7.14 & 22.49 & 14.10 & 21.34 & 12.11 & 20.36 & 11.73 & 9.94 & 8.50 & 12.00 \\
\hline \multirow[t]{3}{*}{ SDNN (ms) } & Holter & 52 & 89 & 168 & 208 & 112 & 113 & 145 & 144 & 130 & 145 \\
\hline & Our & 50 & 85 & 162 & 203 & 103 & 111 & 142 & 136 & 121 & 139 \\
\hline & Relative error (\%) & 3.85 & 4.49 & 3.57 & 2.40 & 8.04 & 1.77 & 2.07 & 5.56 & 6.92 & 4.14 \\
\hline \multirow[t]{3}{*}{ SDANN (ms) } & Holter & 46 & 74 & 157 & 167 & 100 & 107 & 128 & 123 & 130 & 136 \\
\hline & Our & 45 & 72 & 162 & 168 & 101 & 111 & 118 & 128 & 129 & 138 \\
\hline & Relative error (\%) & 2.17 & 2.70 & 3.18 & 0.60 & 1.00 & 3.74 & 7.81 & 4.07 & 0.77 & 1.47 \\
\hline \multirow[t]{3}{*}{ RMSSD (ms) } & Holter & 10 & 24 & 29 & 60 & 24 & 20 & 32 & 32 & 16 & 27 \\
\hline & Our & 11 & 27 & 33 & 57 & 22 & 26 & 33 & 37 & 16 & 32 \\
\hline & Relative error (\%) & 10.00 & 12.50 & 13.79 & 5.00 & 8.33 & 30.00 & 3.13 & 15.63 & 0.00 & 18.52 \\
\hline \multirow[t]{3}{*}{ PNN50 (\%) } & Holter & 0 & 3.2 & 7 & 32.7 & 2.4 & 2.7 & 10.5 & 7.9 & 0.9 & 4.4 \\
\hline & Our & 0.2 & 4.2 & 7.2 & 33.1 & 1.5 & 2.9 & 10.8 & 9.4 & 1.1 & 3.9 \\
\hline & Relative error (\%) & 0.00 & 31.25 & 2.86 & 1.22 & 37.50 & 7.41 & 2.86 & 18.99 & 22.22 & 11.36 \\
\hline \multirow[t]{3}{*}{ \# PAC beats } & Holter & 21 & 3,118 & 19 & 182 & 5 & 40 & 3,015 & 467 & 40 & 754 \\
\hline & Our & 53 & 2,152 & 41 & 355 & 7 & 71 & 2,584 & 425 & 48 & 1,214 \\
\hline & Relative error (\%) & 152.38 & 30.98 & 115.79 & 95.05 & 40.00 & 77.50 & 14.30 & 8.99 & 20.00 & 61.01 \\
\hline \multirow[t]{3}{*}{ \# PVC beats } & Holter & 0 & 16,490 & 0 & 139 & 0 & 16 & 14,437 & 3 & 23 & 4 \\
\hline & Our & 47 & 18,264 & 3 & 172 & 33 & 60 & 14,069 & 97 & 32 & 23 \\
\hline & Relative error (\%) & 0.00 & 10.76 & 0.00 & 23.74 & 0.00 & 275.00 & 2.55 & 3133.33 & 39.13 & 475.00 \\
\hline
\end{tabular}

The mean heart rates were different, but they were within reason according to the total times and total beat
ECGs were comparable to the Holter monitor, while there was large bias for PAC and PVC detection.

\section{Discussion and conclusion}

The ECG signals were recorded synchronously by a Holter and a wearable ECG system, and the standard ECG parameters, derived from the wearable ECG system, were compared with the Holter reports.

Almost all the total recoding times of the wearable ECG system were longer than the Holter, except volunteer 4 and 10 , which reflected that the wearable ECG system was more comfortable than Holter. It is interesting that the total recording times of Holter were shorter than of the wearable ECG system, while the detected beat numbers from Holter were larger than from the wearable ECG system. The reason was that although the dry electrodes used in the wearable ECG system could provide more comfort, it would introduce more noises during daily activities. 
numbers. The slowest heart rate and times of bradycardias were approximate between Holter and the wearable ECG system, which confirmed that the signal quality evaluation step before R-wave peak detection guaranteed the accuracy of rhythm parameter analysis in almost all the volunteers.

For heart rate variability, the SDNN, SDANN and RMSSD fluctuated slightly, indicating the difference were in reasonable variation range. Almost all the PNN50 derived from the wearable ECG system are all larger than from Holter, it is because the presence of noise causes the positioning of the R-wave peak to be biased. However, the difference percentages were all smaller than 1 except volunteer 8 , indicating that the adopted $\mathrm{R}$-wave detection is suitable for wearable ECG signals, and the derived PNN50 is acceptable for long-term HRV analysis.
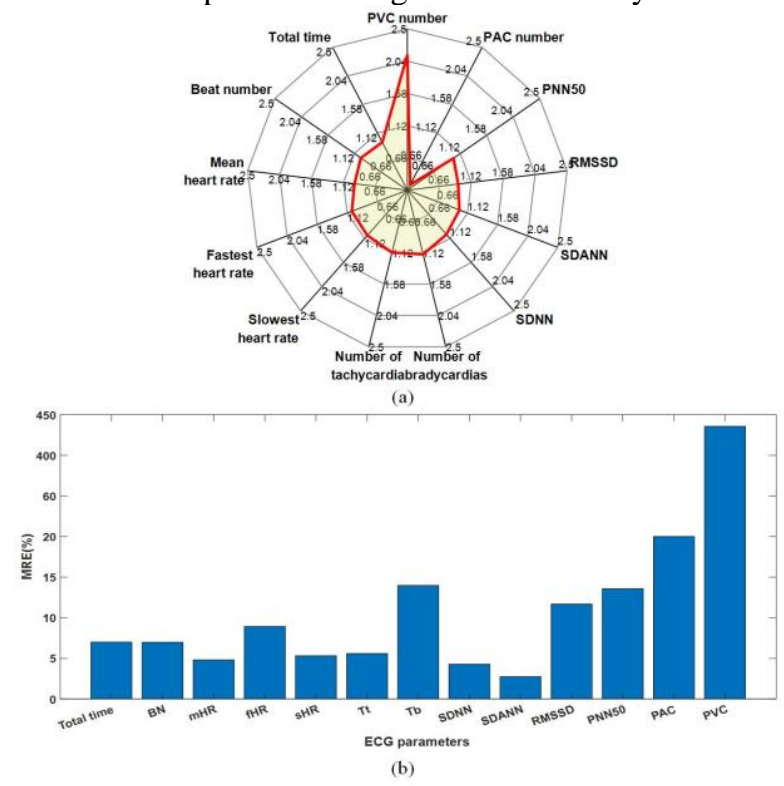

Figure 2 The comparison results between Holter monitor and the wearable ECG system. (a) The ratio of mean values for each ECG parameter, (b) the MREs of the ten volunteers.

The comparison of the ratios of mean values for each ECG parameter shows that the signal quality evaluation step before R-wave peak detection guaranteed the accuracy of detected R-wave peaks, resulting in the rhythm parameter analysis, the R-wave recognition and HRV analysis are comparable to Holter reports. It indicates that the wearable ECG system could not only provide comfort during wearer's daily activity, but also be used for portable ambulatory ECG monitoring for health monitoring and abnormal alarm applications.

\section{Acknowledgments}

The project was partly supported by the National Natural Science Foundation of China (61571113 and 81871444), the Natural Science Foundation of Jiangsu Province (BE2017735).

\section{References}

[1] E. J. Benjamin, S. S. Virani, C. W. Callaway, A. M. Chamberlain, A. R. Chang, S. Cheng, S. E. Chiuve, M. Cushman, F. N. Delling, and R. Deo, "Heart disease and stroke statistics - 2018 update: a report from the American Heart Association," Circulation, vol. 137, pp. e67-e492, Mar. 2018.

[2] C. Liu, X. Zhang, L. Zhao, F. Liu, X. Chen, Y. Yao, and J. Li, "Signal quality assessment and lightweight QRS detection for wearable ECG SmartVest system," IEEE Internet of Things Journal, June. 2018.

[3] Y. Zheng, X. Ding, C. C. Y. Poon, B. P. L. Lo, H. Zhang, X. Zhou, G. Yang, N. Zhao, and Y. Zhang, "Unobtrusive sensing and wearable devices for health informatics," IEEE Transactions on Biomedical Engineering, vol. 61, pp. 15381554, May 2014.

[4] A. Pantelopoulos and N. G. Bourbakis, "A survey on wearable sensor-based systems for health monitoring and prognosis," IEEE Transactions on Systems, Man, and Cybernetics, Part C (Applications and Reviews), vol. 40, pp. 1-12, 2010, Art. no. 5306098.

[5] B. Jeon, J. Lee and J. Choi, "Design and implementation of a wearable ECG system," International Journal of Smart Home, vol. 7, pp. 61-69, 2013.

[6] X. Liu, H. Du, G. Wang, S. Zhou, and H. Zhang, "Automatic diagnosis of premature ventricular contraction based on Lyapunov exponents and LVQ neural network," Computer Methods and Programs in Biomedicine, vol. 122, pp. 47-55, Oct. 2015.

[7] W. K. Lee, H. Yoon and K. S. Park, "Smart ECG monitoring patch with built-in R-peak detection for long-term HRV analysis," Annals of Biomedical Engineering, vol. 44, pp. 2292-2301, Jul. 2016.

[8] C. Chen and C. Chuang, "A QRS detection and R point recognition method for wearable single-lead ECG devices," Sensors, vol. 17, pp. 1969(1)-1969(19), Sep. 2017.

[9] M. Di Rienzo, V. Racca, F. Rizzo, B. Bordoni, G. Parati, P. Castiglioni, P. Meriggi, and M. Ferratini, "Evaluation of a textile-based wearable system for the electrocardiogram monitoring in cardiac patients," Europace, vol. 15, pp. 607612, Apr. 2012.

[10] C. Liu, P. Li, Y. Zhang, Y. Zhang, C. Liu, and S. Wei, "A construction method of personalized ECG template and its application in premature ventricular contraction recognition for ECG mobile phones," in World Congress on Medical Physics and Biomedical Engineering May 26-31, 2012, Beijing, China, 2013, pp. 585-588.

[11] M. Xu, S. Wei, X. Qin, Y. Zhang, and C. Liu, "Rule-based method for morphological classification of ST segment in ECG signals," Journal of Medical and Biological Engineering, vol. 35, pp. 816-823, Dec. 2015.

Address for correspondence:

Jianqing Li and Chengyu Liu

School of Instrument Science and Engineering, Southeast University

ljq@seu.edu.cn and chengyu@seu.edu.cn 